in only one of two ways : either by recruiting and training more medical microbiologists or by concluding that microbiology is not a medical discipline. If that conclusion was accepted all the work would be done by graduates in science and by technicians.

The present examination of these possibilities concludes that medical graduates have clinical skills and a background of pathology extremely useful in the application of microbiology to medicine and that this application, to be fully effective, requires what medical graduates have been trained to give. In promoting the advance of microbiology as a science in its own right medical microbiologists have, perhaps surprisingly, played a significant and useful part. Their recruitment and training need systematic thought about methods and objectives as well as some additional effort and imagination. Emphatically it should be continued.

\title{
Ischaemic Contracture of Muscle Associated with Carbon Monoxide and Barbiturate Poisoning*
}

\author{
A. J. G. HOWSE, $†$ F.R.C.S., D.OBST.R.C.o.g. ; Sir HERBERT SEDDON, C.M.G., M.A., D.M., F.R.C.S., F.A.C.S.(HON.)
}

Brit. med. F., 1966, 1, 192-195

Ischaemic muscle contracture was first described by von Volkmann in 1881, since when the condition has become generally recognized as a complication of certain injuries in the limbs. In a recent review of Volkmann's ischaemia one of us (Seddon, 1964) referred to cases associated with carbon monoxide and barbiturate poisoning, and this paper contains an account of four of them.

Despite the frequency of carbon monoxide poisoning its association with muscle necrosis is little known. References to it in the literature have been few and scattered.

A detailed description of the muscle changes associated with carbon monoxide poisoning was first given by Larrey (1812). $\mathrm{He}$ had the opportunity of examining several cases in troops during the occupation of Berlin by Napoleon in 1806. The soldiers used to heat their quarters by burning coal in open stoves, and the lack of ventilation produced many cases of carbon monoxide poisoning. At necropsy Larrey found brown discoloration of the muscles with softening of the fibres and also skin necrosis over the sacrum and shoulder-blades.

A similar but less precise account of accidental asphyxiation by carbon monoxide in a tavern in Jena is given by Hoffmann (1754) ; he wrote on the subject in 1740, but we have not had access to this earlier work. Knowledge of the gas, as distinct from carbon dioxide, existed before Priestley described it in 1772 and Dalton deduced its formula in 1808.

The legs of the Jena victims were probably affected by gangrene, similar to that later described by McLean (1911), Briggs (1919), and Fowler (1954).

It might well be thought that muscle necrosis due to carbon monoxide poisoning is rare; however, this is probably by no means so. Petri (1930) suspected that it was not, though he reported only one case; Gunther $(1921,1940)$ reported nine, three of his own; Hedinger (1948) reported two cases.

Necrosis of muscle, indistinguishable from that caused by traumatic ischaemia, can occur in patients who have taken an overdose of a barbiturate, and in view of the widespread addiction to these drugs this condition may be worthy of wider recognition. We put these two kinds of muscle ischaemia together because, as is explained later, we regard their aetiology as almost identical ; in brief, hypoxaemia and external pressure, the latter of a degree that would be tolerable in a normal person.

We are reporting four further cases of ischaemic necrosis of muscle followed by contracture ; two followed coal-gas poisoning and two barbiturate overdosage.

\section{Case 1}

On 20 September 1952 a man of 30 lost consciousness when about to boil some water on a gas-stove. He was alleged to have burned the dorsum of his left hand and the flexor surface of the right forearm. He was treated for carbon monoxide poisoning and the so-called burns. On recovering consciousness he noted: (1) the fingers of the right hand were flexed-a similar contracture on the left followed about eight weeks later ; (2) numbness of the palmar surfaces of the hands and fingers, which subsequently passed off ; (3) hardness of the flexor muscles of both forearms, which largely disappeared during the next eight weeks.

The initial treatment was by corrective splints and mobilization; he attended a rehabilitation centre with some further improvement, and then returned to work. However, the contractures became a nuisance and he was admitted to the Royal National Orthopaedic Hospital on 17 February 1953.

Right Upper Limb.-There were two very superficial scars on the flexor surface of the forearm. Excessive sweating was present over the ulnar side of the palm; the radial side and the radial three digits were dry and cold. The brachial pulse was normal and the radial pulse nearly so. There was a flexion contracture of the long flexors of the thumb, and of the index, middle, and ring fingers, so that the fingers could be fully extended only when the wrist was in the neutral position. Hypoaesthesia was present over the middle and distal phalanges on the palmar aspect of the radial three digits. Tinel's sign was positive at the wrist over both median and ulnar nerves.

Left Upper Limb.-The flexor muscles of the forearm were hard, especially about $10 \mathrm{~cm}$. above the wrist on the ulnar side; severe contracture of the long flexors of all the digits was present, so that the finger-tips were about $1.5 \mathrm{~cm}$. from the palm when the wrist was in the neutral position; the thumb was flexed $90^{\circ}$ at the interphalangeal joint and $45^{\circ}$ at the metacarpophalangeal joint. On flexion of the wrist all but the middle finger could be fully extended. Dorsiflexion of the wrist was limited to $45^{\circ}$, and the hand deviated to the ulnar side. There was also an adduction contracture of the thumb and wasting of all intrinsic muscles of the hand. There was paralysis of the thenar muscles. Hypoaesthesia was present over the palmar surface of the distal two phalanges of the fingers, and Tinel's sign was positive over the median and ulnar nerves at the wrist.

Operation.-Correction of flexion contracture on left side was carried out on 19 February. An arteriogram showed nothing more than slight loss of the fine arterial branches in the distal quarter of the forearm.

* From the Institute of Orthopaedics, Great Portland Street, London.

+ Senior Orthopaedic Registrar, Middlesex Hospital, London. Now Consultant Orthopedic Surgeon at Central Middlesex, Willesden General, and Wembley Hospitals. 
Muscles.-There was diffuse fibrosis from the deep fascia down to the bones. Flexor digitorum sublimis was fibrotic over a length of about $6 \mathrm{~cm}$., and in ftexor digitorum profundus there was intense fibrosis throughout the whole thickness of the muscle from 7 to $14 \mathrm{~cm}$. above the wrist with a patch of necrosis laterally, the muscle being avascular, yellowish green, and friable. On the lateral side of this necrotic patch was a curious bursa with a whitish fibrotic wall separating it from a similar patch of necrosis in the medial part of the belly of the flexor pollicis longus. Flexor digitorum profundus was grossly shortened, especially the portion destined for the middle finger. Flexor pollicis, longus was also shortened. Both of these muscles were firmly adherent to the underlying bone and to the pronator quadratus. The latter also appeared ischaemic but was not explored.

Nerves. - The median nerve was narrowed to about two-thirds of its normal diameter between 9 and $13 \mathrm{~cm}$. above the wrist, and this part was abnormally soft. A small branch, probably the palmar branch, arose just above the zone of narrowing and was removed for histological examination. Electrical stimulation produced no response. The ulnar nerve appeared normal, and on stimulation there was a contraction in the hypothenar muscles, the medial lumbricals, adductor pollicis, and flexor pollicis brevis. A minute specimen was removed for section from a segment opposite the zone of greatest damage.

Procedure.-The flexor digitorum profundus was divided completely, and this corrected about half the deformity of the fingers. This left a strong elastic resistance from the flexor digitorum sublimis, which it was thought could be corrected by appropriate splinting. A Z-shaped lengthening of the flexor pollicis longus was carried out with division of the adhesions between it and the radius; full correction of this contracture was obtained.

The operation was followed by active exercises. By September 1953 the right hand was virtually normal, and by March 1955 there was a full range of passive movements in both hands.

Histology. - The small branch of the median nerve showed severe ischaemic collagenization and few nerve fibres. The ulnar nerve showed less severe involvement ; numerous slender myelinated nerve fibres, certainly regenerating, were present (Fig. 1). Flexor pollicis longus and flexor digitorum profundus showed complete necrosis. Flexor digitorum sublimis showed necrosis with a small area of less severe damage in which there was shrinkage of the muscle fibres and much interstitial fibrosis.

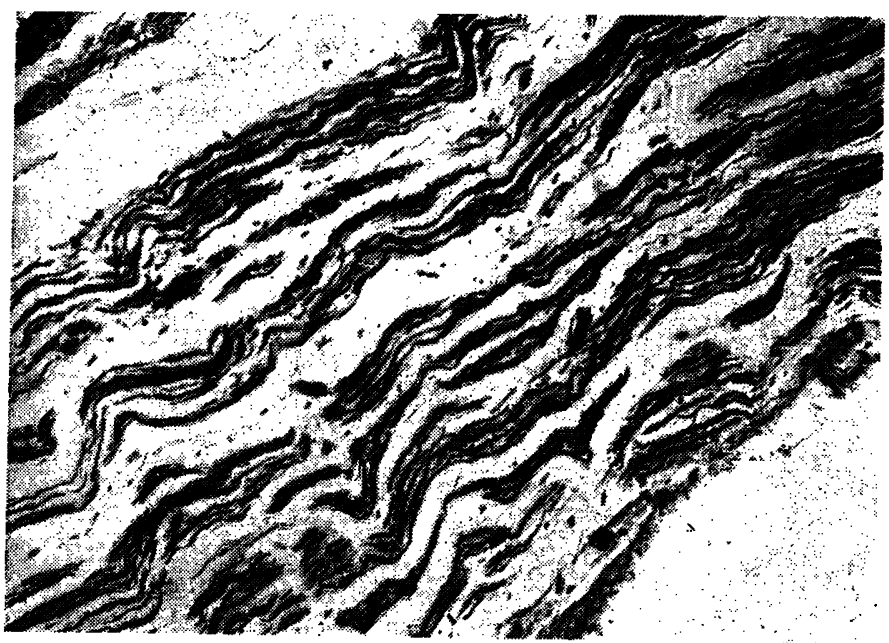

FIG. 1.-Regenerating nerve fibres in a branch of the ulnar nerve (Case 1).

\section{Case 2}

On 15 December 1958 a woman of 43 was found unconscious as a result of barbiturate poisoning; she had been lying on the right forearm and hand. On recovery of consciousness she felt extreme pain in the forearm and hand, and there was complete paralysis below the elbow. The only treatment she received was elevation of the limb. Five months later there was blistering of the skin with ulceration along the proximal third of the ulna.

When seen in September 1959 she was still complaining of pain in the hand, restricted extension of the wrist, and weakness of extension of the fingers. She thought that the range of these movements was decreasing.

A mobile painless scar, $12.5 \mathrm{~cm}$. long, was present over the proximal third of the ulna. The wrist was held in radial deviation, the metacarpo-phalangeal joints were held in the neutral position, and the proximal interphalangeal joints were flexed to a right-angle. No supination of the forearm was possible, but movement at the elbow was full. The wrist could not be extended, but it flexed $30^{\circ}$. The metacarpo-phalangeal joints of the fingers flexed only $30^{\circ}$. She had an adductor contracture of the thumb and a flexor tendon contracture of the interphalangeal joint of $10^{\circ}$. She had between $20^{\circ}$ and $40^{\circ}$ loss of straightening of the proximal interphalangeal joints of the fingers but the distal joints were almost fully mobile. Voluntary power was almost normal in the forearm apart from slight weakness of the radial extensors of the wrist, inability to extend the fingers against gravity, and paralysis of the long extensor of the thumb. The small muscles of the hand were paralysed. Light touch was appreciated as far distally as the metacarpo-phalangeal joints. Pin-prick was appreciated in the median area to the metacarpo-phalangeal joints but not at all in the ulnar area.

She was admitted to the Royal National Orthopaedic Hospital in November 1959. Her condition had deteriorated since her outpatient visit.

Operation.-Forearm muscle slide for correction of the contracture was carried out on 2 December. There was necrotic muscle in the deeper part of the forearm flexor mass, and it was excised. The muscles were detached from their origins and the ulnar nerve was transplanted anteriorly. The nerves were not explored.

By February 1960 there had been some improvement in sensibility in that light touch could be appreciated in the median area to the middle of the intermediate phalanges of the fingers and over the whole of the thumb. Pain was appreciated to the tip of the little finger.

By October 1960 movements of the fingers were almost full No flexion was possible at the wrist-joint, though it could be extended to $45^{\circ}$. There was hypersensitivity of the palm and fingers severe enough to compel the patient to wear a glove for protection.

By 1965 the pain in the hand had gradually diminished and good though not perfect sensibility had returned. The long flexors of the fingers were weak, the long flexor of the thumb was paralysed, and the extensors of the fingers were extremely weak. The intrinsic muscles of the hand had recovered well. The significant contractures were total absence of pronation and $55^{\circ}$ lack of extension of the metacarpo-phalangeal joints of the fingers.

\section{Case 3}

A man of 39 was admitted unconscious to hospital on 23 March 1962, having been found in front of a gas-fire turned on but unlit. Examination revealed cherry-red mucous membranes, Cheyne-Stokes respiration, and extensive blistering of the right hand around the thenar area with much oedema at this site and of the forearm. His general condition improved with oxygen.

On 4 April he developed paralysis of the left ulnar, median, and posterior interosseous nerves. Despite treatment he developed contractures of both hands. He was admitted to the Royal National Orthopaedic Hospital on 30 April.

Right Upper Limb. - There was a five-degree flexion contracture of the proximal interphalangeal joints of the index and middle fingers, hard swelling of the thenar eminence, and in the first web space limited movement of the thumb. The fingers could be flexed. There was loss of sensibility in the median area.

Left $U_{p p e r}$ Limb.-There was contracture of all the long flexors of the digits, and the fingers were clawed. The fingers could be flexed through about three-quarters of their normal range. The small muscles of the hand were paralysed and sensibility was lost in the median and ulnar areas. There was induration of the forearm at the junction of the proximal and middle thirds which rapidly decreased after intensive physiotherapy and occupational therapy.

Operation.-On 26 May the following were carried out : (1) release of the contracture of the right thumb and biopsy of the muscles-they were greyish brown and did not respond to electrical stimulation; and (2) the left forearm flexors and extensors were 
explored. A band of pallor in the flexor muscles was found $15-23 \mathrm{~cm}$. below the medial epicondyle, and there was pallor and narrowing of the median nerve in this region. On the extensor aspect there was a similar zone of abnormality $9-17 \mathrm{~cm}$. below the lateral epicondyle. No other procedure was considered necessary.

The contractures on the left side slowly decreased, and by September 1962 full passive movement was possible ; sensory loss persisted distal to the mid-palm. However, the right thumb became fixed in such extreme opposition as to prevent flexion of the index finger.

The patient was readmitted to hospital on 13 August 1963 He had a satisfactory grip in the left hand, although fine movements and pinch grip were a little impaired; sensibility was diminished in the median and ulnar areas. The right thumb was almost fixed in adduction (Fig. 2).

Operation.-On 15 August the right thenar muscles, including the adductor and first dorsal interosseous, were excised completely. They were densely fibrotic, with yellow patches here and there An arthrodesis of the first carpo-metacarpal joint was performed.

Histology.-All specimens showed ischaemic changes in varying degree, the nerves as well as the muscles being affected.

The result of the operation was satisfactory, but the patient committed suicide in February 1965.

\section{Case 4}

On 20 April 1964 a woman of 36 became deeply unconscious after taking $600 \mathrm{mg}$. of sodium amytal, although previous doses of up to $1 \mathrm{~g}$. had not had that effect. She had not apparently taken alcohol as well. After recovering in hospital she noticed a red raw area just above her right wrist. This was thought to be and was treated as a burn. One week later she could not fully straighten her thumb and fingers, and this contracture increased despite splintage. The hand also became painful and burning, and felt as if it had been blown up. At first the hand was insensitive, but this improved.

She habitually slept on her right side, with the volar surface of the forearm uppermost.

She was admitted to the Royal National Orthopaedic Hospital on 8 September 1964 . The interphalangeal joints of all the right digits were held acutely flexed but without hyperextension of the metacarpo-phalangeal joints (Fig. 3). Pinkish discoloration of the skin was seen over the lower third of the volar surface of the right forearm. There was firm induration of the flexor muscles between 10 and $18 \mathrm{~cm}$. below the elbow.

Flexion of the wrist was $70^{\circ}$ and extension $45^{\circ}$. Supination was full, but pronation only $70^{\circ}$. The metacarpo-phalangeal joints were normal. The interphalangeal joint of the thumb lacked $70^{\circ}$ of extension; the proximal interphalangeal joints of the fingers all lacked about $30^{\circ}$ of extension and the distal interphalangeal joints about $20^{\circ}$.

The flexor-tendon contracture was such that the fingers could be held nearly straight only with the wrist flexed $65^{\circ}$. All the intrinsic muscles of the hand showed extreme wasting. In the median area sensibility was lost down to the mid-palm. There was over-reaction to pin-prick in the whole ulnar area. The median area was dry, the ulnar area moist. The thenar muscles and interossei were paralysed and the hypothenars very weak.

Operation.-Forearm muscle slide to correct the contracture was carried out on 10 September. An ellipsoid mass of pallid fibrotic muscle extended from 3 to $9 \mathrm{~cm}$. above the wrist, involving flexor pollicis longus, flexors digitorum sublimis and profundus, and the proximal third of pronator quadratus. The median nerve was of normal size but slightly firmer than usual and firmly adherent to the long flexors from 6 to $9 \mathrm{~cm}$. above the wrist. The muscles were detached from their origins, and this corrected about half the deformity; it decreased a little subsequently.

She made a very good recovery (Fig. 4), and by mid-1965 the hand was almost normal.

Histology.-Sections showed atrophic muscle fibres embedded in dense fibrous tissue and very occasional necrotic fibres, but no large mass of necrotic muscle. The appearances were those of the periphery of an infarct.

\section{Discussion}

The initiating factor is tissue anoxia and the subsequent changes can affect all structures. At first the patient may complain of great pain in the affected parts, which swell greatly. At about the same time the skin becomes red,

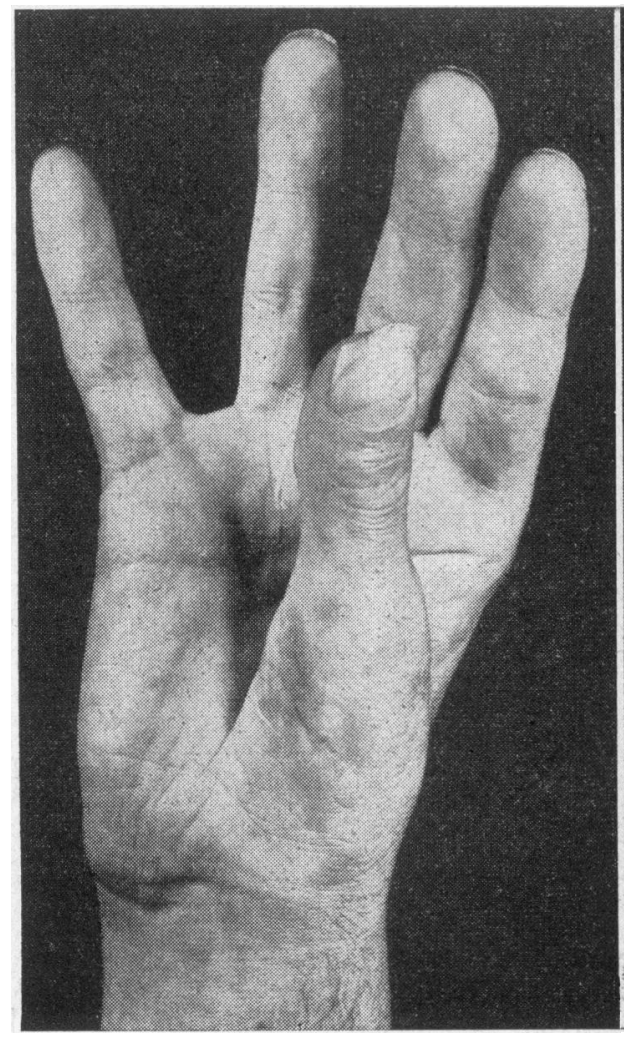

FIG. 2

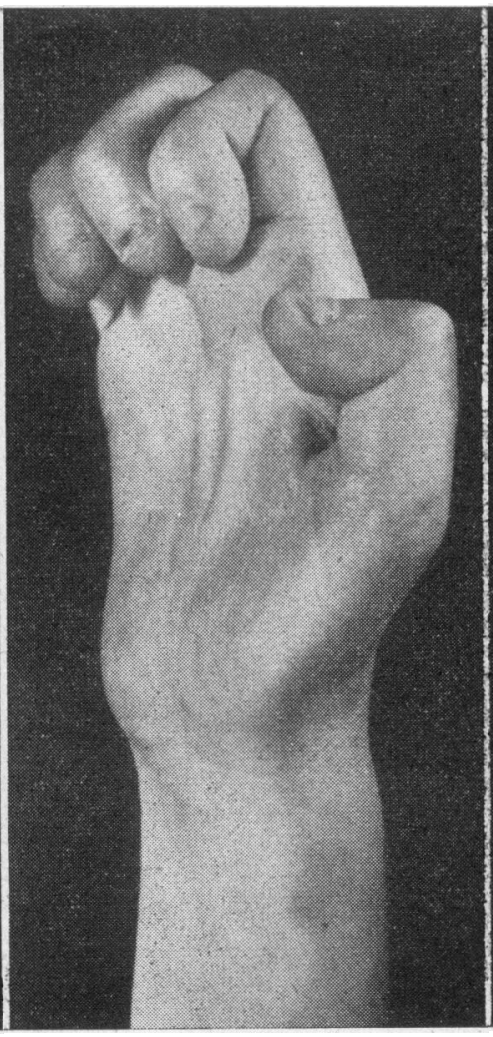

FIG. 3

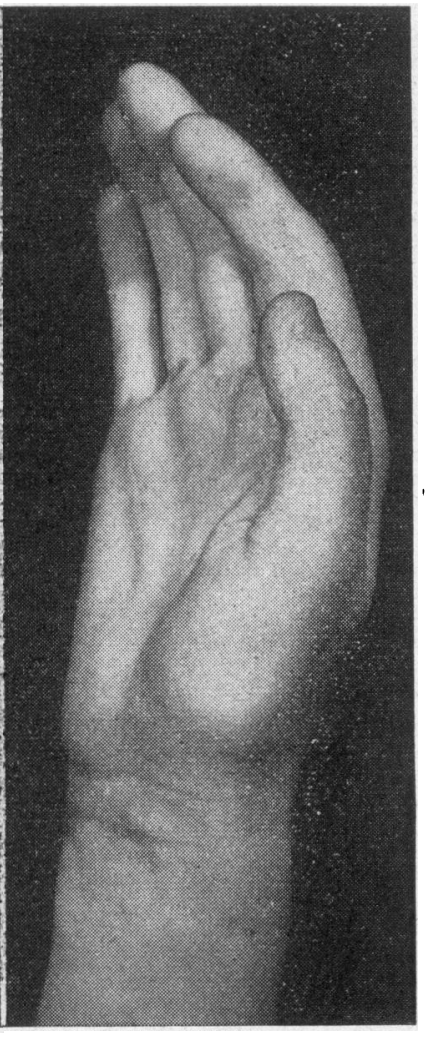

FIG. 4

Fig. 2.-Right thumb in Case 3. Adduction contracture and wasting of the first cleft. Fig. 3.-Contracture in Case 4. FIG. 4. - Condition of hand in Case 4 three months after operation. 
blistered, and sometimes even necrotic, though these changes may be delayed for a considerable period (Case 2). Motor and sensory paralysis develops either early (Cases 1 and 2) or later (Case 3). Subsequently hardening of the affected muscles occurs, followed by contracture. The damaged nerves usually recover, though perhaps imperfectly.

The tissue damage is indistinguishable from that found in Volkmann's ischaemia. Indeed, the only distinctive feature is the tendency for the ischaemic areas to be sharply localized, multiple, and unrelated to the distribution of any particular artery, as in Case 3, where there was a band of fibrosis in the forearm and fibrosis of the thenar muscles.

Myocardial infarction as a complication of carbon monoxide poisoning has been recognized for some time (Groetschel, 1940 ; Leathart, 1963) and regarded as due to anoxaemia. In skeletal muscle there is an additional factor. From a study of the sites affected by the ischaemia and of the positions in which the patients were lying when unconscious we have come to the conclusion that external pressure is as important as the anoxaemia (Fig. 5). Scharmann (1921) and Hedinger (1948) also found that muscle necrosis occurred at sites of pressure, as had been suggested by Larrey's (1812) cases.

F. A. Ducharme (personal communication) believes that the ischaemia is a result of swelling beneath the deep fascia, which then strangles the enclosed tissues. However, this would not explain the sharply localized areas in our cases. The pressure would be about equal throughout a mass of tissue enclosed within one fascial envelope, the soft parts within it behaving rather like a liquid. Furthermore, certain masses of tissue, such as the thenar muscles, have no fascial envelope.

It is conceivable that in some cases the muscle-and-nerve ischaemia could be due mainly to vascular occlusion, though we think this unlikely. There are few references to this in the literature, though necrosis of arteriolar walls with thrombosis has been reported in carbon monoxide poisoning by Loughridge, Leader, and Bowen (1958).

General hypoxia must render tissues liable to total anoxia if they are also subjected to external pressure, if only of a degree that would have no effect on the muscles or nerves of a healthy person. So far as we have been able to discover Volkmann's ischaemia is not found in patients after prolonged periods of unconsciousness from causes other than poisoning, such as cerebrovascular accidents. However, Adams, Denny-Brown, and Pearson (1962) have observed ischaemic lesions in the heart and limb muscles of persons who have survived for 24 to 48 hours after suffering from prolonged anoxaemia or circulatory collapse, though this is not quite comparable with the unconsciousness following poisoning. In carbon monoxide poisoning the anoxia is due to the depression of respiration and to the combination of the carbon monoxide with haemoglobin to the exclusion of oxygen. Leathart (1963) also stresses the

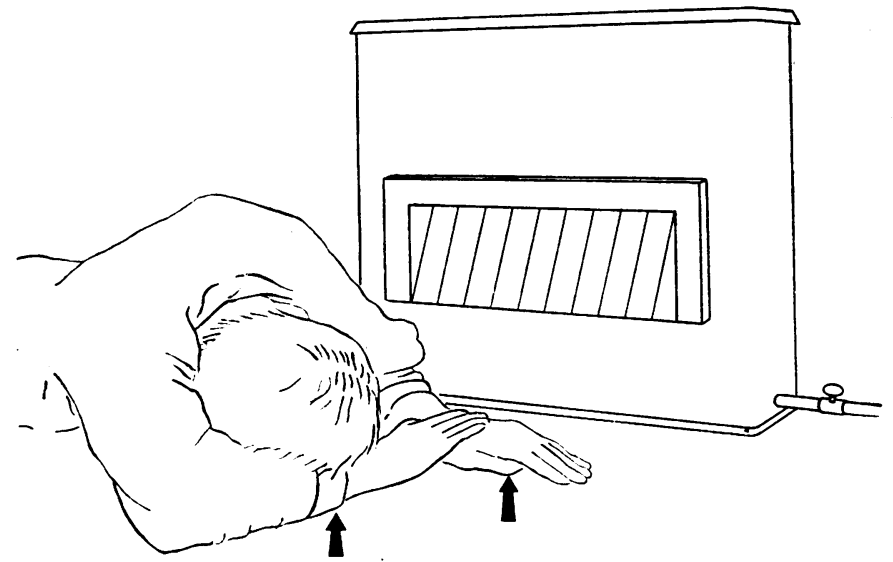

FIG. 5.-Position in which the patient was found (Case 3). Arrows indicate the points of pressure, which correspond with the sites of ischaemia. importance, not previously considered, of the combination of the carbon monoxide with oxygen-carrying substances-for example, myoglobin-in the tissues themselves. He believes this can lead to chronic carbon monoxide poisoning, the occurrence of which is denied in this country, but not on the Continent. Chronic carbon monoxide poisoning might explain the delayed onset of some of the changes; in Case 3, for example, there was a 12-day delay before the wrist-drop appeared. Adams et al. (1962) state that the combination of carbon monoxide with myoglobin renders muscle more susceptible to ischaemia caused by pressure. In barbiturate poisoning the respiratory depression is very profound; thus the anoxia from this factor alone is likely to be greater than in carbon monoxide poisoning. Graham (1962) believes that prolonged and severe hypoxia is the cause of the tissue changes in both barbiturate and carbon monoxide poisoning. Beveridge and Lawson (1965) regard barbiturates as directly responsible for the cutaneous lesions they observed in cases of poisoning ; they dismiss the possible role of pressure and ignore the muscle changes we describe.

In recent years muscle necrosis has been reported after various types of general intoxication: alcohol, alcohol and hypnotics, or hypnotics alone (Jasiński and Brütsch, 1952; Touzé and Thierry, 1952; Fahlgren, Hed, and Lundmark, 1957) and general anaesthesia (Gordon and Newman, 1953).

An important complication of the muscle necrosis, which fortunately did not occur in our cases, is renal damage and even renal failure as a result of blockage of the tubules by the breakdown products of the muscle necrosis similar to that occurring in the crush syndrome. Gunther (1921) and Loughridge et al. (1958) each report a fatal case, and Mautner (1955) reports one with temporary oliguria ; all followed carbon monoxide poisoning.

\section{Summary}

Four cases of ischaemic muscle contracture associated with carbon monoxide or barbiturate poisoning are reported. It is probable that the condition is more common than the number of previously reported cases suggests.

Ischaemic nerve lesions occur, as in the more usual traumatic Volkmann's ischaemia.

The cause of this condition is almost certainly a combination of local pressure and general hypoxia.

We wish to thank Mr. Donal Brooks for permission to report one of his cases.

\section{REFERENCES}

Adams, R. D., Denny-Brown, D., and Pearson, C. M. (1962). Disease of Muscle, 2nd ed. Kimpton, London.

Beveridge, G. W., and Lawson, A. A. H. (1965). Brit. med. F., 1, 835

Briggs, J. E. (1919). F. Amer. med. Ass., 73, 678.

Fahlgren, H., Hed, R., and Lundmark, C. (1957). Acta med. scand., 158,405 .

Fowler, P. B. S. (1954). Lancet, 1, 240.

Gordon, B. S., and Newman, W. (1953). F. Bone ft Surg., 35A, 764

Graham, J. D. P. (1962). The Diagnosis and. Treatment of Acute Poisoning. Oxford Univ. Press, London.

Groetschel, H. (1940). Arch. Gewerbepath. Gewerbehyg., 10, 223.

Gunther, H. (1921). Z. klin. Med., 92, 41

(1940). Ergebn. inn. Med. Kinderheilk., 58, 331.

Hedinger, C. (1948). Schweiz. med. W schr., 78, 145. Hoffmann, F. (1754). Operum Omnium Physico-Medicorum, Supplement,
2nd ed. Fratres de Tournes, Geneva.

Jasiński, B., and Brütsch, H. (1952). Schweiz. med. Wschr., 82, 29.

Larrey, D. J. (1812). Mémoires de Chirurgie Militaire et Campagnes, 3, 13. Smith and Buisson, Paris.

Leathart, G. L. (1963). Trans. Ass. industr. med. Offrs, 13, 96.

Loughridge, L. W., Leader, L. P., and Bowen, D. A. L. (1958). Lancet,

2, 349. (1911). f. Amer. med. Ass., 56, 1455.

Mautner, L. S. (1955). Arch. Path., 60, 136.

Petri, E. (1930). "Kohlenoxyd," in Pathologische Anatomie und Histologie der Vergiftungen, Bd. 10, Handbuch der speziellen pathologischen Anatomie und Histologie, edited by F. Henke and $O$. Lubarsch.

Scharmann, G. (1921). Dtsch. Arch. klin. Med., 135, 336

Seddon, H. J. (1964). Brit. med. F., 1, 1587.

Touzé, J., and Thierry, M. (1952). Bull. Soc. méd. Hôp. Paris, 68, 971. Volkmann, R. von (188h). Zbl. Chir., 8, 801. 